\title{
Use of Nonlinear Optics for Assessment of Cable Polymer Aging
}

\author{
Kaylee N. Rellaford ${ }^{1}$, Dallin L. Smith ${ }^{1}$, Alexander J. Farnsworth ${ }^{1}$, Shane M. Drake ${ }^{1}$, Hoon Lee ${ }^{1}$, and James E. Patterson ${ }^{1}$ \\ ${ }^{1}$ Department of Chemistry and Biochemistry, Brigham Young University, Provo, UT, 84602, USA \\ jepatterson@chem.byu.edu
}

\begin{abstract}
Polymer jackets play an important protective role in distribution cabling by providing structure and resistance to moisture, heat, and exposure to harmful chemicals. Current methods of structural assessment, such as elongation at break (E-at-B), are inherently destructive. While other nondestructive methods such as indenter evaluation are available, they are not suitable for in-service use. We propose that second harmonic generation (SHG) could provide a nondestructive means of characterizing the aging of chlorosulfonated polyethylene (CSPE) cable jackets. SHG was used to study cables previously aged and characterized by the Electric Power Research Institute (EPRI). Comparative data between the SHG results and indenter modulus tests suggest that SHG can be used to qualitatively differentiate between minimally and significantly aged CSPE cable jackets. The results of this proof-of-concept study suggest additional work that could be done to better understand the mechanisms of the aging of CSPE cable jackets and how SHG could be used to monitor the aging process.
\end{abstract}

\section{INTRODUCTION}

Much of modern technology and society depends on the safe and reliable distribution of electric power from the point of generation to the point of consumption. Polymer jackets are used to protect the underlying distribution cable core from mechanical deformation, moisture penetration, and chemical damage during the installation and service life of the cable. Jackets also enhance flame resistance, facilitate installation, and provide means for cable identification and grouping (USA Wire \& Cable). As the polymer jacket ages, especially if it is subjected to mechanical stress and/or harsh conditions, such as extremes or changes of temperature, the cross-linking structure of the polymer can rearrange and therefore the jacket can become more susceptible to cracking (Verardi, 2014). Cracking, as well as other effects of aging, can

Kaylee Rellaford et al. This is an open-access article distributed under the terms of the Creative Commons Attribution 3.0 United States License, which permits unrestricted use, distribution, and reproduction in any medium, provided the original author and source are credited.

https://doi.org/10.36001/IJPHM.2021.v12i2.2966 compromise the protective nature of the polymer jacket and is the leading indicator of insulation degradation. Such exposure of the insulation to the outside environment would warrant additional attention and potential replacement.

One way to assess aging of a polymer jacket is by elongation at break (E-at-B) measurements. The inherently destructive nature of this test, however, prohibits its use on cables currently in service. Other non-destructive, visual/tactile tests are used in the field, such as the bending test and indenter evaluation, however these can also have limitations for cables that are in service (EPRI \& DOE, 2005). A non-destructive evaluation (NDE) method to detect aging that could be used without repositioning or modifying the cables in any way would be beneficial in determining the remaining usable life of distribution cables. The following work evaluates the use of a nonlinear optical method, second harmonic generation (SHG), as a means of characterizing the state of polymer cable jackets that have been subjected to artificial aging. The work was performed on three sets of cables with jackets made using chlorosulfonated polyethylene (CSPE), also known as Hypalon $^{\mathrm{TM}}$, from three different manufacturers/models: Rockbestos Firewall III, Okonite, and BIW cable systems. (Hypalon is the trademark name of Dupont's CSPE material.) These cables had been aged and characterized previously by the Electric Power Research Institute (EPRI). The optical measurements are here correlated with the EPRI E-at-B and indenter modulus tests. This proof-of-concept study shows that SHG has potential as a method for initial screening of aged cable jackets, although more work will need to be done before SHG can be deployed as a NDE platform for the monitoring of distribution cables.

\section{Methods}

\subsection{Thermal Aging}

The three cable systems were: Rockbestos Firewall III cables (CSPE jacket with cross-linked polyolefin insulation), Okonite cables (CSPE jacket with ethylene propylene rubber insulation), and BIW Cable Systems (CSPE jacket with ethylene propylene rubber insulation). Aging conditions were established by EPRI using Arrhenius based accelerated 
thermal aging to simulate natural aging at various stages of operating time in power plants. The cables were spaced out on racks in a convection oven for thermal aging, with sufficient spacing to allow for appropriate air circulation. All cables were initially thermally aged at $119^{\circ} \mathrm{C}$ for various periods of time, as listed in Table 1. Following the initial aging, additional aging was performed on select BIW and Rockbestos cables to simulate severe aging. This aging was performed at $124^{\circ} \mathrm{C}$ for the specified time. Further details on the thermal aging protocols can be found in EPRI technical report No. 1001391 (EPRI \& DOE, 2002).

\subsection{SHG Measurements}

The light source used for generating a second-harmonic response was a pulsed $532 \mathrm{~nm}$ frequency doubled Nd:YAG laser (Minilite II, Continuum). A basic diagram of the SHG setup is shown in Figure 1. The laser operated at a $10 \mathrm{~Hz}$ rep rate with a pulse width of 3-5 ns. Average laser power at the sample was $15.1 \mathrm{~mW}$ (15.1 $\mathrm{mJ}$ per pulse), and the laser was focused to a spot size of approximately $2.5 \mathrm{~mm}$ diameter at the sample. Prior to the focusing lens, the beam passes through a waveplate that is used to optimize the polarization for maximum SHG signal. The second-harmonic signal, which occurs at $266 \mathrm{~nm}$, was reflected off two commercial dichroic harmonic separators to separate the signal from residual $532 \mathrm{~nm}$ pump light and passed through additional UV filters before detection by a photomultiplier tube (PMT) that was connected to a digital oscilloscope. Insertion of a glass slide before the PMT allowed for detection of the residual $532 \mathrm{~nm}$ light; the glass absorbed the $266 \mathrm{~nm}$ SHG light. The time-dependent signal from the PMT was captured for each shot of the laser (Rellaford, Averett, Farnsworth, Adams, Smith, Fullwood \& Patterson, 2021).

Due to the relatively large spot size of the probe laser, a single measurement interrogates multiple microstructures in the polymer. To obtain a representative measurement of the cable, each sample was scanned 5 times at locations spaced approximately $5 \mathrm{~mm}$ apart. Three sets of measurements were taken across the length of each sample. All samples were scanned in as-received condition; no surface modification or preparation was done prior to the SHG measurements.

A typical SHG measurement involves illuminating the sample for 80-100 shots of the laser at each spot. Half of these shots were taken without the glass slide in the optical path and include both SHG and background light. The second half was taken with the glass slide inserted to measure only the background light. Typical traces from the PMT, both with and without the glass slide, are shown in Figure 2. Each signal trace was separately integrated over time with a trapezoidal integration to determine the total signal for that laser shot. The integrated values were then averaged for each of the two sets (signal+background and background only), and the two averaged values were subtracted to produce a single value for the SHG response from that location. These values were then averaged for multiple spots to provide a value that is representative of the entire cable specimen.

\begin{tabular}{|c|c|c|c|}
\hline Material & $\begin{array}{l}\text { Aging } \\
\text { Step }\end{array}$ & $\begin{array}{c}\text { Aging (hours) } \\
\text { at } 119^{\circ} \mathrm{C}\end{array}$ & $\begin{array}{c}\text { Aging (hours) } \\
\text { at } 124^{\circ} \mathrm{C}\end{array}$ \\
\hline \multirow[t]{4}{*}{ BIW Cable } & 1 & 780 & - \\
\hline & 2 & 1559 & - \\
\hline & 3 & 2278 & 384 \\
\hline & 4 & 3042 & 400 \\
\hline \multirow{4}{*}{$\begin{array}{l}\text { Okonite } \\
\text { Cable }\end{array}$} & 1 & 780 & - \\
\hline & 2 & 1441 & - \\
\hline & 3 & 2278 & - \\
\hline & 4 & 3042 & - \\
\hline \multirow{4}{*}{$\begin{array}{l}\text { Rockbestos } \\
\text { Cable }\end{array}$} & 1 & 780 & - \\
\hline & 2 & 1441 & - \\
\hline & 3 & 2278 & - \\
\hline & 4 & 3042 & 400 \\
\hline
\end{tabular}

Table 1. Thermal aging of specimens. Adapted from EPRI report No. 1001391 (2002).

\section{RESULTS}

\subsection{Previous EPRI Measurements}

As documented in EPRI Report No. 1008211, the elongation at break (E-at-B) of Hypalon cable jackets decreases as the sample ages (EPRI \& DOE, 2005). A representative response is shown in Figure 3. (The behavior of additional materials is shown in the EPRI report.) This behavior, and other effects such as dielectric breakdown, is caused by the stiffening of the material with age, typically due to changes in the crosslinking of the rubber polymer (Bowler, 2020; Takahashi). While E-at-B is widely accepted as a method for estimating life of materials such as Hypalon, it cannot be used on material that is intended to remain in service, because the method is inherently destructive.

Other testing methods that are less invasive can be used to evaluate remaining life of cables, such as a bend test and nanoindentation. The data in Figure 4 show the nanoindentation test results (completed by EPRI and more fully described in EPRI technical report No. 1001391, 2002) 


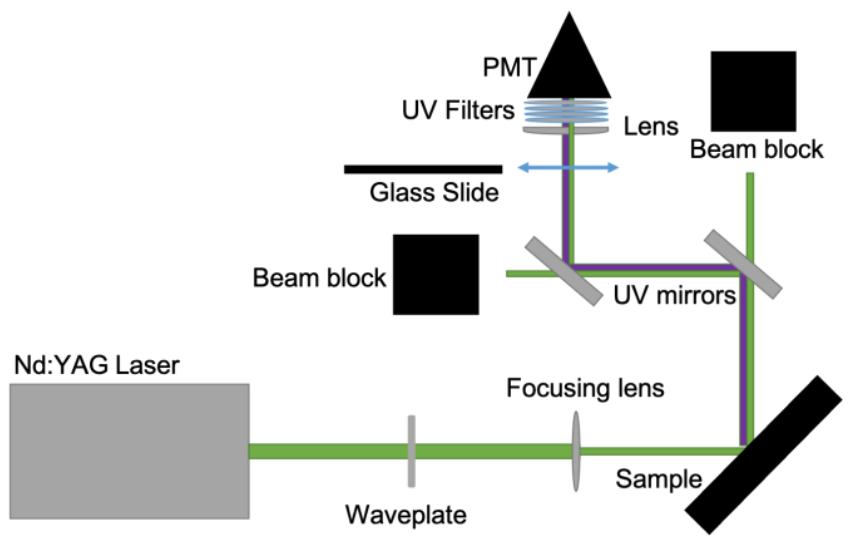

Figure 1. SHG experimental setup. The glass slide is inserted into the beam path for background measurements.

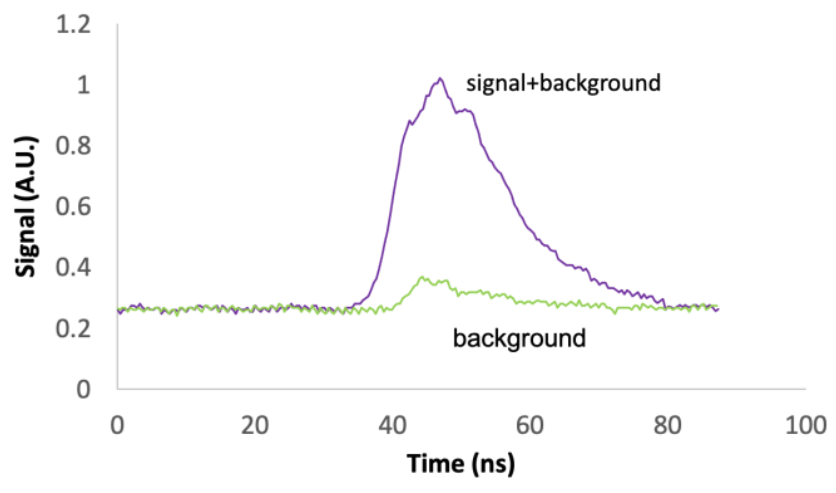

Figure 2. Representative traces of PMT signal for signal+background (upper) and background only (lower).

for the 3 different cable sets analyzed in this study: Rockbestos Firewall III, Okonite, and BIW Cable Systems, respectively.

For all three cable systems, the indenter modulus increased with aging, indicating that the polymer had become stiffer. The change is fairly gradual with aging steps for the Rockbestos Firewall III cables, whereas the Okonite and BIW Cable Systems samples showed a much more dramatic increase in the indenter modulus following the second aging step. The BIW Cable System specimens that were subjected to additional aging showed even greater increases in indenter modulus. Even though all three cable jackets are nominally the same CSPE material, methods of compounding the jacket vary for each manufacturer, which results in different aging behavior.

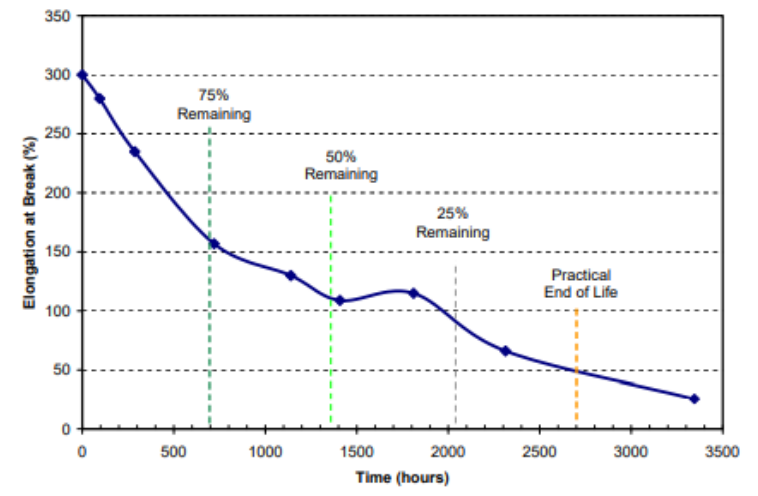

Figure 3. Remaining life curve based on elongation at break (E-at-B) measurements for CSPE that had been artificially aged at $110^{\circ} \mathrm{C}$ (EPRI \& DOE, 2005).

\subsection{SHG Measurements}

SHG measurements were performed on the same sets of Hypalon $^{\mathrm{TM}}$ cables from Figure 4: Rockbestos Firewall III, Okonite, and BIW Cable Systems. Scans were collected from multiple locations on each cable in the individual sets, with the results from a single cable averaged to report a value for that cable specimen. The SHG results for the same cables are shown in Figure 5.

For all three Hypalon ${ }^{\mathrm{TM}}$ jacketed cable types, the amount of SHG response decreased with aging, however the pattern across the stages of aging is different for each type of cable. For the Rockbestos Firewall III cables, the SHG signal generally decreased with each aging stage, although there was a significant increase in SHG signal for the $3^{\text {rd }}$ aging stage. This probably merits further investigation. The Okonite cables showed a roughly linear decrease in SHG signal for the first two aging stages, but then a significant decrease for the $3^{\text {rd }}$ and $4^{\text {th }}$ aging stages. The behavior of both the Rockbestos Firewall III and Okonite cables bears strong resemblance to the trend seen in the E-at-B measurements, as shown in Figure 3. The BIW cables showed a dramatic decrease in SHG signal even after the first aging stage, and this signal never returned with additional aging.

It should be noted that we did find that there was a small amount of damage caused by the intense laser light at the sample surface. If the laser power was decreased to avoid surface damage, no SHG signal was produced. However, the surface damage does not affect the performance of the cable jacket and did not affect our results. In a future st udy, it should be possible to identify operational conditions that can generate a meaningful SHG response without scarring the sample, but that was beyond the scope of this proof-ofconcept study. 

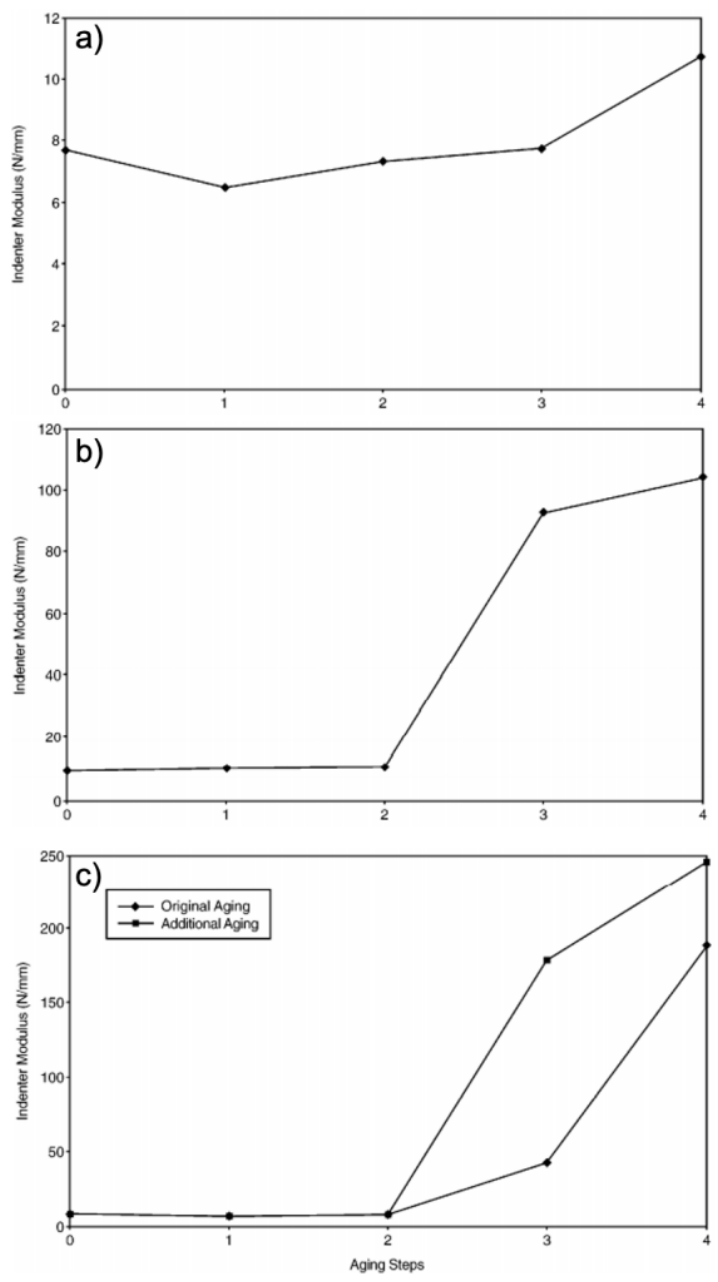

Figure 4. Indenter modulus measurements versus aging steps for a) Rockbestos Firewall III cables, b) Okonite cables, and c) BIW Cable System cables (EPRI \& DOE 2002.)

\section{DISCUSSION}

\subsection{Comparison of Indenter and SHG Measurements}

The results presented in Section 3 measure the degree of aging via two different methods, indenter modulus and SHG response. Data in Figure 4, from EPRI, show a general increase in indenter modulus with aging for each Hypalon sample. The data in Figure 5 show a general decrease in SHG signal with aging for the same samples. To aid the reader in comparing the trends, Figure 6 shows the SHG data overlaid on the EPRI indenter modulus data for each cable system. Note that the $\mathrm{y}$-axis for SHG signal is inverted to enable better comparison of the trends. Figures $6 a$ ) and $6 \mathrm{~b}$ ) show a strong agreement between the two methods for evaluating aging. For the Rockbestos Firewall III cables (Fig. 6a), the indenter modulus increases gradually, with some variation,
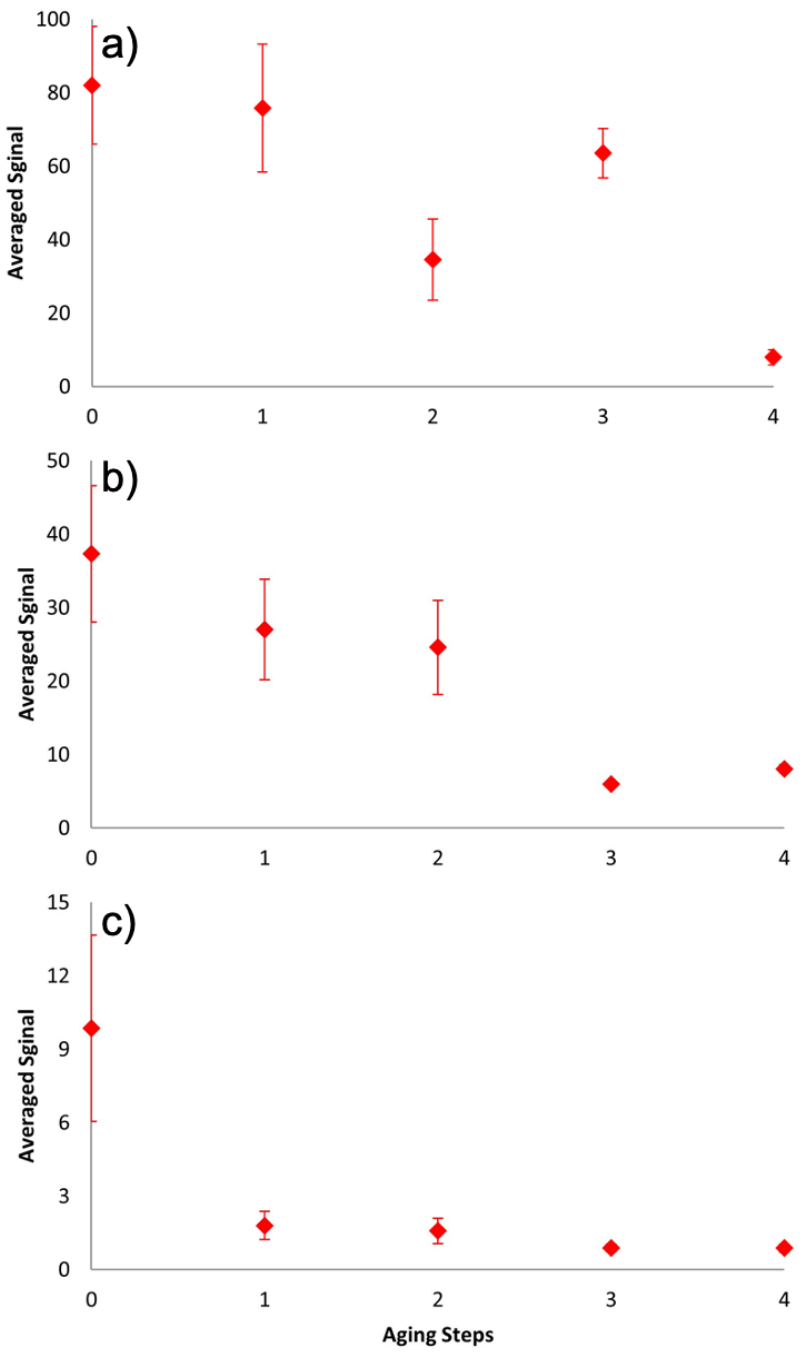

Figure 5. SHG response average for all spots across the sample for a) Rockbestos Firewall III cables, b) Okonite cables, and c) BIW Cable System cables.

with aging. Likewise, the SHG signal tends to decrease, again with some variation. The Okonite cables (Fig. 6b) show little change up through the second aging step, then a dramatic increase in indenter modulus, with a similar decrease in SHG response, upon the third and fourth aging steps.

A weaker correlation between the two measurements exists for the BIW cables, as seen in Figure 6c. While the first and last aging step match closely, the methods do not seem to correspond in the intermediate range. This may result from the BIW cables having undergone additional heating beyond what the Rockbestos and Okonite cables received (EPRI \& DOE, 2002). Consequently, the BIW cables exhibited a yellowish-brown surface coating that EPRI researchers described as "bloom." To determine the impact of this phenomenon on the SHG measurements, scans were conducted on areas with and without bloom on the surface, 

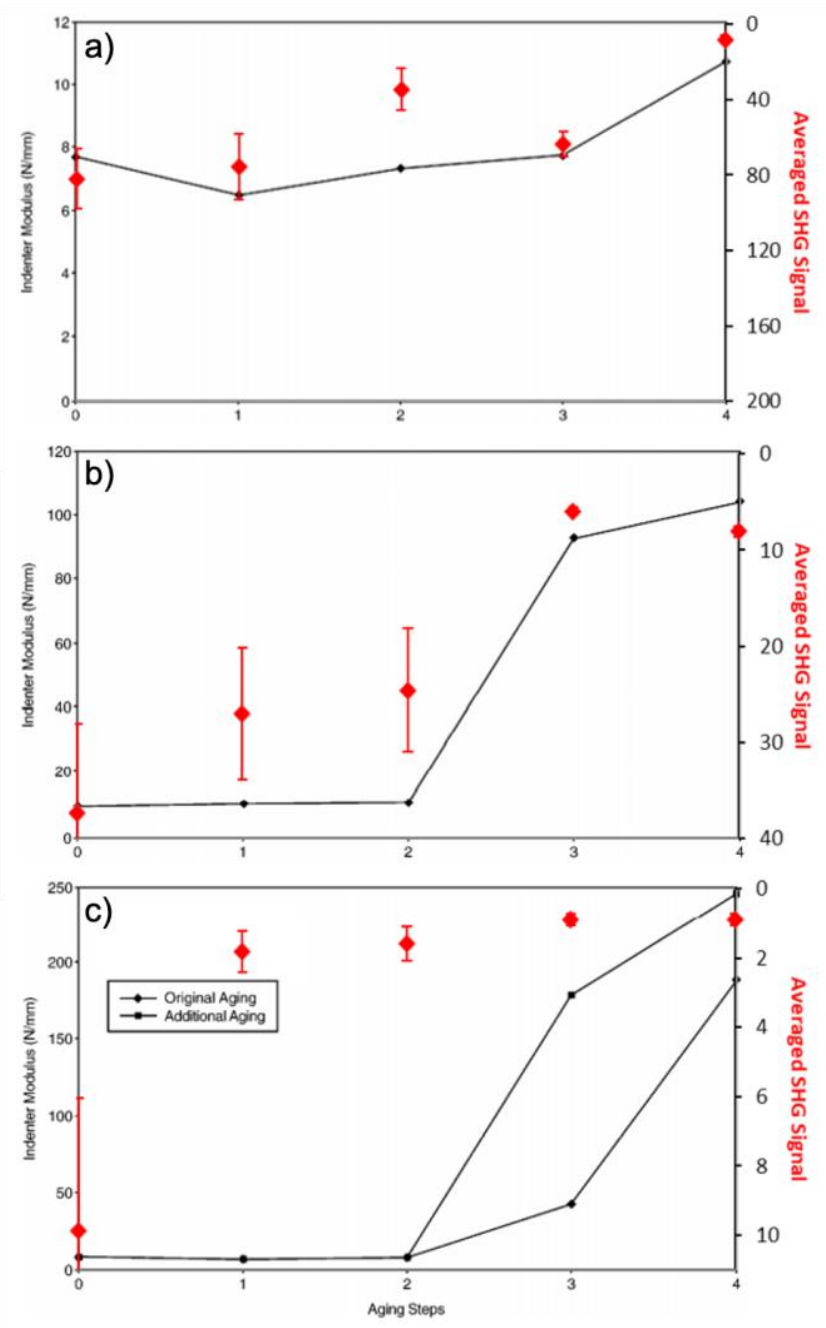

Figure 6. SHG response (large red markers) overlaid with indenter results (small black markers) for a) Rockbestos Firewall III cables, b) Okonite cables, and c) BIW Cable System cables. The axis for SHG signal is inverted from what was shown in Fig. 5 to aid comparison.

with no significant difference between them. The data reported here were collected on areas with no bloom.

\subsection{Possible Mechanisms of Aging}

CSPE (Hypalon ${ }^{\mathrm{TM}}$ ) and many other thermoset polymers owe much of their industrial utility to the crosslinks that form between the polymer chains. Artificial aging, however, has been shown to cause chain scission, which, along with thermal activation, allows for greater molecular mobility and structural relaxation. This resultant relaxation, or annealing, can lead to secondary crystallization, which can increase the overall crystallinity of the material and may rearrange molecules at the interface of amorphous-crystalline regions (White, 2006; Wang, Ni, Bian, Zhang \& Zhang, 2010). The rate of secondary crystallization is depth-dependent; the rate is higher at the surface because of the ready availability of oxygen. Below the surface, the rate depends on the oxygen diffusion and consumption rates of the material (Boukezzi, Boubakeur, \& Lallouani, 2007). Another consequence of chain scission, formation of new crosslinks, and secondary crystallization is increased embrittlement of the polymer.

Both indenture tests and SHG probe the sample surface, and the results of these measurements can be used to infer structural changes to the material that result from aging. The E-at-B and indentation data from EPRI show evidence of embrittlement as a result of aging. The dramatic decrease in elongation at break for heavily aged samples is a clear sign of the loss of flexibility of the polymer. For all three cable systems, successive steps of aging consistently lead to a higher indentation modulus, indicating that a greater force is required for the material to yield; the material has become stiffer and more brittle. To the extent that the SHG measurement correlates with these indentation tests, SHG could also be used to assess aging and the resultant increase in brittleness of CSPE cable jackets.

The central requirement of SHG is that the signal can only be generated from a region that lacks inversion symmetry. The most obvious location that satisfies this condition is the surface of the polymer. There are, however, two other locations where the symmetry can be broken in such a way as to produce a SHG response. These are at the boundaries of crystalline and amorphous domains within the bulk of the sample, and within crystalline domains themselves, provided those crystalline domains belong to non-centrosymmetric space groups.

Thermoset polymers form complex semi-crystalline structures with large portions of ordered regions and sections of amorphous polymer chains. This semi-crystalline nature makes classifying the space groups for a material difficult (Sperling, 2001). While we could not readily find a reported space group for CSPE crystals, one of the stable or metastable crystalline structures may be centrosymmetric. For example, high-density polyethylene (HDPE) is known to form crystalline regions that belong to the centrosymmetric $P_{n m a}$ space group (Bunn, 1939). Other crosslinked polymer crystal structures have been characterized, some of which are also centrosymmetric (Saito, Nansai, \& Kinoshita, 1972; Soldatov, Suwinska, Terekhova, \& Manakov, 2008). Formation of similar domains in CSPE would mean no SHG signal would be generated from within those crystalline domains. One mechanism, therefore, by which the SHG signal could be reduced due to aging would be a conversion of crystalline domains from non-centrosymmetric to centrosymmetric space groups.

The interface between crystalline and amorphous domains is another potential source of SHG signal. As discussed in our previous work on HDPE, however, such regions are likely not a major contributor to the measured signal. When SHG is used to characterize a buried interface, typically there is a significant difference in one or more properties across that 
interface, such as density, optical index of refraction, or chemical identity (Averett, Stanley, Hanson, Smith, \& Patterson, 2018). For the amorphous/crystalline boundary within bulk CSPE, none of these properties will differ significantly on either side of the interface. This leaves the surface as the most likely source of the SHG response.

Crystallite restructuring is not the only process that has been reported to affect an aging polymer. Chain scission and monomer mobility could also lead to structural disorder without secondary crystallization. This would mean that a sample's regular and semi-ordered surface could become disrupted and more randomized. The resulting increase in azimuthal symmetry at the surface would lead to a decreased SHG susceptibility, and therefore less SHG signal generation.

Based on these preliminary measurements, we propose that surface order disruption and/or secondary recrystallization from a more SHG susceptible space group to a less susceptible one is the molecular mechanism by which the SHG response decreases upon sufficient aging. While we cannot currently determine which of these processes dominates the response of CSPE to aging, these results help set the stage for further investigation. Such studies would require additional controlled aging, with monitoring of the samples before, during, and after the aging process. Additional techniques such as X-ray diffraction (XRD) and electron microscopy would likely provide additional insight to the aging process.

\section{CONCLUSION AND FUture WORK}

This initial, proof-of-concept study shows that SHG is a promising non-destructive technique for investigating thermal aging of CSPE cable jackets. SHG can distinguish between samples with minimal aging and samples with significant aging; the data in section 4.1 show that the difference in SHG signal is statistically significant regardless of cable manufacturer, and it aligns well with the indentation results. In each case, SHG response was minimal at stage 4 of aging, which coincides with maximum indenter modulus. However, the intermediate range of thermal aging is more difficult to quantify with SHG than differentiating between unaged and highly aged samples. Additional studies would be needed to fully ascertain the capability of SHG to identify samples that have only been aged to an intermediate degree.

Development of SHG into a full NDE platform will require additional work to pair the SHG analysis with complementary measurements, such as indentation modulus and crystallinity. Such a concerted effort will reveal more details about the aging process for CSPE polymers. As explained above, one hypothesized cause for decreasing SHG signal is an increase in non-centrosymmetric secondary crystallinity caused by thermal aging. Overall, this study shows that SHG has potential to provide supplementary information as an NDE platform for various brands of
Hypalon (CSPE) cable jackets. While the proof-of-concept results presented herein are promising, much remains to be done.

\section{ACKNOWLEDGEMENT}

This work was supported, in part, by the Electric Power Research Institute (EPRI), under Agreement 10010491. The authors also thank Andrew (Drew) Mantey of EPRI for providing the cable samples and important input on this manuscript.

\section{REFERENCES}

Averett, S. C., Stanley, S. K., Hanson, J. J., Smith, S. J., \& Patterson, J. E. (2018). Surface spectroscopic signatures of mechanical deformation in high-density polyethylene (HDPE). Applied Spectroscopy, 72(7), 1057-1068. doi:10.1177/0003702818757232

Boukezzi, L., Boubakeur, A., \& Lallouani, M. (2007). Effect of artificial thermal aging on the crystallinity of XLPE insulation cables: X-ray study. 2007 Annual Report Conference on Electrical Insulation and Dielectric Phenomena, 65-68. https://doi.org/10.1109/CEIDP.2007.4451517

Bowler, N.; Liu, S. (2015) Aging Mechanisms and Monitoring of Cable Polymers. International Journal of Prognostics and Health Management, 6 (3), 2287. doi:10.36001/ijphm.2015.v6i3.2287

Bunn, C. W. (1939). The crystal structure of long-chain normal paraffin hydrocarbons. The "shape" of the $<\mathrm{CH} 2$ group. Transactions of the Faraday Society, 35, 482491. doi:10.1039/TF9393500482

Electric Power Research Institute and U.S. Department of Energy. (2002). Training aids for visual/tactile inspection of electrical cables for detection of aging (Report No. 1001391). Retrieved from https://www.epri.com/research/products/1001391.

Electric Power Research Institute and U.S. Department of Energy. (2005). Initial acceptance criteria concepts and data for assessing longevity of low-voltage cable insulations and jackets (Report No. 1008211). Retrieved from https://www.epri.com/research/products/1008211.

Rellaford, K. N., Averett, S. C., Farnsworth, A. J., Adams, D. D., Smith, S. D., Fullwood, D. T., \& Patterson, J. E. (2021) Characterization of mechanical deformation in aluminum by optical second harmonic generation. Measurement Science and Technology, 32, 075202. doi: 10.1088/1361-6501/abe668

Saito, Y., Nansai, S., \& Kinoshita, S. (1972). Structural studies on polyurethane fibers. Polymer Journal, 3, 113121. doi:10.1295/polymj.3.113

Soldatov, D. V., Suwinska, K., Terekhova, I. S., \& Manakov, A. Yu. (2008). Structural investigation of hydrate compounds of the tetraisoamylammonium of polyacrylate ion exchange resins. Journal of Structural 
Chemistry, 49, 712-718. doi:10.1007/s10947-008-0099$\mathrm{z}$

Sperling, L. H. (2001). Introduction to physical polymer science (3rd ed.). John Wiley and Sons Ltd.

Takahashi, T.; Ohtsuka, H.; Takehana, H.; Niwa, T. Influence of insulation morphology, impurities and oxidation on some electric properties of cables. https://ieeexplore.ieee.org/stamp/stamp.jsp?tp=\&arnum ber $=68234 \& \operatorname{tag}=1$ (accessed Jun 16, 2021).

USA Wire \& Cable, Inc. (n.d.). Wire Knowledge. https://www.usawirecable.com/pdfs/wire\%20knowledge.pdf

Verardi, L., Fabiani, D., \& Montanari, G. C. (2014). Electrical aging markers for EPR-based low voltage cable insulation wiring of nuclear power plants. Radiation Physics and Chemistry, 94, 166-170. doi:10.1016/j.radphyschem.2013.05.038

Wang, Z., Ni, H., Bian, Y., Zhang, M., \& Zhang, H. (2010). The preparation and thermodynamic behaviors of chlorosulfonated polyethylene. Journal of Applied Polymer Science, 116(4), 2095-2100. doi:10.1002/app.31726

White, J. R. (2006). Polymer ageing: physics, chemistry or engineering? Time to reflect. Comptes Rendus Chimie, 9(11), 1396-1408. doi:10.1016/j.crci.2006.07.008 